\title{
COMMUNICABLE DISEASES REPORT, MARCH AND APRIL 2005
}

For updated information, including data and facts on specific diseases, visit www.health.nsw.gov.au and click on Infectious Diseases.

\section{TRENDS}

Tables 2 and 3 and Figure 1 show reports of communicable diseases received through to the end of March and April 2005 in NSW.

Reports of cases of the mosquito-borne Ross River virus (59 cases) and Barmah Forest virus (34 cases) infections increased in March, mainly in the North Coast and Hunter/ New England Areas.

A rise in case reports of laboratory-confirmed mumps was identified earlier in 2005 (15 cases in January, 12 cases in February) and affected mainly men and women in their twenties in the Northern Sydney/Central Coast Area. Reports of cases declined to six in March and seven in April.

A large increase in reported cases of malaria (94 cases) occurred in March. This was largely due to screening of newly arrived refugees from Africa who settled in the Sydney West, Sydney South West and Hunter/New England Areas. These are all imported cases as the Anopheles mosquito required to transmit malaria does not survive in NSW.

\section{ENTERIC DISEASE}

Reports of diarrhoea due to giardiasis (151 cases) and shigellosis (11 cases) were elevated in March, although cases were scattered across several areas of the State and no common source for the outbreaks has been identified. The number of notifications of cryptosporidiosis continued to increase in March (41 cases) and April (69 cases) compared to the same period in 2004, signifying a later rise than usual for the summer period spanning 2004-2005 than for previous years. No common source outbreaks have been identified.

There have been 236 cases of salmonellosis caused by infection with Salmonella Typhimurium phage type 170/108 (STM 170/108) reported in NSW since midOctober 2004. A state-wide case-control study to identify the cause commenced in March. Eighty-three patients with $S$. Typhimurium and 49 disease-free individuals were interviewed. The aim of the study is to determine whether certain dietary risk factors are associated with STM170/108 infection. NSW Health is working with the NSW Food Authority to follow up any common food sources that are identified through the study.

The investigation into a community-wide outbreak of salmonellosis caused by infection with Salmonella Typhimurium phage type 197 continued, with 31 cases notified during February and March. Twenty-three patients were interviewed. The majority (19) were of Lebanese descent and a case-control study to identify the likely cause of the outbreak is underway.

An investigation commenced into an increase in the number of cases of Salmonella Reading (10 cases) in April, a rare serovar in NSW and Australia generally. Half of the patients lived in an area on the North Coast of NSW. No common links have been identified among patients.

\section{PERTUSSIS}

Pertussis remains a common cause of serious illness in the community. In babies symptoms can be severe (with the classic triad of chronic paroxysmal coughing, post tussive vomiting and inspiratory whooping), and in adults symptoms are generally milder (mainly an irritating chronic cough). Cases are infectious from the prodrome to up to three weeks after onset of the cough. The infection is spread by respiratory droplets, and close contacts (especially household members) are at risk of infection. While cases are presenting across the State, the number of notifications of pertussis has continued to fall since peaking in September 2004 (see: http://www.health. nsw.gov.au/data/diseases/pertussis.html). Several notable incidents have been reported in recent weeks and these are described below.

\section{A pertussis case}

The Hunter Population Health Unit reported that in early April 2005 it was notified that a health care worker who worked in obstetrics had been diagnosed with pertussis. The patient's symptoms-paroxysmal, non-productive cough without vomiting or an inspiratory whoop-had begun in late March 2005 and the patient had consulted a doctor a few days later. The doctor had taken a nasopharyngeal swab to test for pertussis using a polymerase chain reaction assay. The person had worked for several days before the diagnosis of pertussis was made, and therefore whilst infectious. The Hunter Population Health Unit recommended follow-up of those exposed. Twenty-one mothers and 22 babies were identified as contacts and advised to receive antibiotic prophylaxis to prevent infection. Three community contacts were identified and one of these was advised to have antibiotic prophylaxis because of a pre-existing chronic respiratory illness.

\section{Cluster 1}

The Penrith office of the Sydney West Area Health Service Centre for Population Health reported a case of pertussis in a health care worker at a local hospital. The worker reported a coughing illness lasting several weeks before medical attention was sought. The person had been working while infectious. The centre wrote to the parents of all babies born during the period the worker was infectoius, advising them to watch for and report any signs and symptoms of pertussis. One of the mothers reported suffering from a cough for approximately two weeks and was immediately tested for pertussis. The test was positive. Her baby had been in the neonatal intensive care unit for approximately five weeks since birth and the mother had visited the unit 
regularly. Although the mother had been wearing a mask for most of the visits to the unit's nursery, prophylaxis was recommended for all infants in the unit exposed to the mother while she was infectious. Letters were written to families advising them to take their newborn infant to their doctor for antibiotics. No further cases of pertussis were identified among the infants in, or parents visiting, the neonatal intensive care unit. However, three of the mother's extended family members and a second healthcare worker at the hospital were subsequently diagnosed with pertussis. Alerts issued by the infection control staff at the hospital allowed prompt diagnosis and treatment of this case.

\section{Cluster 2}

The South Eastern Sydney and Illawarra Area Health Service Public Health Unit's Sydney office reported two pertussis clusters in maternity units during February and March.

In February the unit was notified of an obstetric worker who had become ill with pertussis at the end of January. Over the subsequent month, further confirmed cases were identified, including three staff members of the facility and two patients: an infant and a mother. The baby most likely acquired infection from the mother, as the baby developed symptoms approximately one month after discharge from the facility. Of interest, a healthy woman potentially exposed in hospital was tested by her family doctor and found to be positive for Bordetella IgA, suggestive of asymptomatic pertussis infection. The hospital was advised to write to patient contacts regarding the need for chemoprophylaxis and observation, and inform their medical attendants; staff were educated through in-service talks about pertussis and offered prophylaxis if appropriate, and the hospital brought forward its staff diphtheria-tetanus-pertussis vaccination program. Staff at the unit also telephoned patient contacts who had already been discharged to alert them to the need for prophylaxis and the significance of symptoms in mothers and babies. In all, as part of the public health response the this cluster, approximately 190 patients who were considered to be potentially exposed were provided with information in writing and/or by telephone. Of these, about 160 individuals were advised to take prophylactic antibiotics.

\section{Cluster 3}

The same public health unit office was notified in midMarch of two cases of confirmed pertussis related to a second obstetric unit, one in an infant born in the unit and one in an obstetric worker. These cases did not have contact with each other. The ensuing investigation identified two further confirmed staff cases and one in an antenatal clinic patient, all with onset in February or March. The occupational health and public health responses were similar to those actions taken in response to cluster 2. Information was provided to approximately 110 patient contacts and prophylactic antibiotics were recommended to approximately 30 patient contacts.

\section{A pertussis outbreak}

The Northern Sydney Public Health Unit reported an outbreak of pertussis in a local health care facility. Since January 2005, 10 cases in staff and 30 in residents of this facility had been notified. On advice from the public health unit, the facility implemented control measures (including rapid identification, testing and isolation of suspected cases) and the number of new cases declined. However, a small number of new cases in staff and patients were subsequently reported and it was suggested that vaccination might be helpful in preventing further cases. There is no evidence for the role of adult pertussis vaccination in the control of outbreaks, and the available formulation (a combined diphtheria-tetanus-pertussis booster vaccine) is relatively new and expensive. However, following discussions with the public health unit and the Communicable Diseases Branch of the NSW Department of Health, the facility decided to offer vaccination to susceptible patients and staff. As a result, 74 patients and 101 staff were vaccinated. The success of the intervention in controlling the outbreak is being evaluated.

\section{Comment}

These clusters and outbreaks illustrate how easily pertussis can spread among high-risk individuals. Public health interventions are aimed at controlling the spread of pertussis. These include maximising routine immunisation of the community in early childhood (at ages two, four and six months, with boosters at four years and in high school), and outbreak control. Outbreak control includes early identification and reporting to the local public health unit of possible cases, and the counselling and treatment of contacts at high risk of developing complications. Health care workers, particularly those who work with children, should be immunised in accordance with NSW Health's policy directive Occupational Screening and Vaccination Against Infectious Diseases (PD2005_338). Other people who regularly deal with young children, including new parents and childcare workers, should also be immunised against pertussis. Clinicians should report suspected cases to, and work with, their local public health unit to help identify close contacts at risk for severe disease (especially children aged under one year old, or other infants who are not fulling immunised), or who could transmit infection to these groups (for example pregnant women and susceptible people in child care centres).

\section{REVISED TUBERCULOSIS GUIDELINES}

NSW Health recently released three revised policy directives and one policy guideline relating to the provision of tuberculosis services. The Tuberculosis Contact Tracing (PD2005_581) policy directive has been revised and includes new advice related to the timing of contact tracing and notification of contacts, use of the Public Health Act, and procedures for screening exposed airline passengers. (See: www.health.nsw.gov.au/policies/ PD/2005/PD2005_581.html.) The Tuberculin Skin Testing 
(TST) (PD2005_580) policy directive has been revised to include information on composition, safety, dosage, storage, administration, consent, indications, interpretation, boosting, and use of alternative tests for the identification of infection with tuberculosis. (See: www.health.nsw.gov. au/policies/PD/2005/PD2005_580.html.)

The Charging for Tuberculosis Related Services (PD2005_ $579)$ policy directive has been revised to clarify issues around visa screening, referral to private providers, payment of Medicare benefits, services provided to detainees in immigration detention centres and the financial responsibility for people on an employer-sponsored long term business entry visa. (See: www.health.nsw.gov.au/ policies/PD/2005/PD2005_579.html.) The Tuberculosis in Children and Adolescents (GL2005_060) guideline has been revised in light of current evidence regarding risk of infection and infectivity, and provides guidance on the diagnosis, prevention and treatment of tuberculosis in children and adolescents. (See: www.health.nsw.gov. au/policies/gl/2005/GL2005_060.html.)

\section{OUTBREAK OF ENTEROVIRAL MENINGITIS, SOUTH-EASTERN SYDNEY, FEBRUARY 2005}

\section{Apo Demirkol, Mark J Ferson, Keira Morgan} South Eastern Sydney Public Health Unit

On 22 February 2005, a local public school contacted the South Eastern Sydney Public Health Unit to seek advice concerning the occurrence of two cases of meningitis among children in the same class. Initial enquiries revealed that around the same time, several students from the same public school had been admitted to Sydney Children's Hospital with suspected viral meningitis. It was decided to mount a public health response to determine whether an outbreak was occurring and, if so, its extent and aetiology.

\section{Investigation}

Since this was an investigation of a non-notifiable condition, we contacted local emergency departments to inform them of the investigation and to seek their collaboration in reporting new cases. In consultation with South Eastern Area Laboratory Services microbiologists, we asked clinicians to request the following tests on new cases: enterovirus polymerase chain reaction assay (PCR) and viral culture on cerebrospinal fluid (CSF) and faeces. In order to investigate possible common exposures, we developed a questionnaire seeking information on acute illness in the household and involvement in recreational water activities within the previous two weeks. A visit was made to the school that made the initial report to assess the toilets and handwashing facilities, and to inform and reassure the staff.

\section{Results}

Active case finding during the three weeks after the initial report revealed 16 cases of viral meningitis in children aged one month to nine years, five of whom attended the same primary school, and one in a young adult aged 20. All the patients lived in Sydney's eastern and southern suburbs. Onset dates for their illnesses ranged from 21 February to 4 March. All bacterial cultures were negative, and enterovirus infection was confirmed by PCR in three cases on CSF samples and in one case on stool. Echovirus was isolated from the CSF of one of these cases. A questionnaire was completed for 13 cases. Fever followed by headache were universal symptoms, whilst seven (54 per cent) experienced neck stiffness. Four (31 per cent) cases denied recent recreational water use, and there were no common recreational water exposures in the remaining nine cases. Seven (54 per cent) had had at least one family member who had been acutely ill within the previous two weeks. Information was provided to the school that had made the initial enquiry about the illness. Inspection of the school's facilities found that cleaning of the children's toilets and washbasins could be improved, and this was brought to the attention of the principal. School staff put significant effort into encouraging the children to use good personal hygiene procedures.

\section{Comment}

Enteroviruses, composed of more than 70 serotypes, circulate predominantly in summer and autumn. They may cause asymptomatic infection and diverse illnesses, including hand, food and mouth disease, influenza-like illness, gastroenteritis and viral meningitis. ${ }^{1}$ Transmission is generally from person-to-person by the faecal-oral route. However, outbreaks of enterovirus infection have been attributed to sewage contamination of drinking or recreational waters. ${ }^{2}$ As common environmental exposures were ruled out, we worked on the hypothesis that a strain of enterovirus was circulating in the community and causing a range of illnesses, including meningitis. In the absence of a common source, it was decided to cease active surveillance. Information was provided to other local schools to reassure staff and parents of the relatively benign nature of the infection and to highlight the role of good hygiene in preventing spread.

\section{References}

1. Sawyer MH. Enterovirus infections: diagnosis and treatment, Pediatr Infect Dis J 1999; 18:1033-40.

2. Gosbell I, Robinson D, Chant K, Crone S. Outbreak of echovirus 30 meningitis in Wingecarribee Shire, New South Wales. Commun Dis Intell 2000, 24:12-124. 월 
FIGURE 1

REPORTS OF SELECTED COMMUNICABLE DISEASES, NSW, FEB 1999 TO APRIL 2005, BY MONTH OF ONSET

Preliminary data: case counts in recent months may increase because of reporting delays.

Laboratory-confirmed cases only, except for measles, meningococcal disease and pertussis $\mathrm{BFV}=$ Barmah Forest virus infections

$\mathrm{RRV}=$ Ross River virus infections

lab $+=$ laboratory confirmed
Men Gp C and Gp B = meningococcal disease due to serogroup $C$ and serogroup $B$ infection, other/unk = other or unknown serogroups.

NB: multiple series in graphs are stacked, except gastroenteritis outbreaks.

NB: Outbreaks are more likely to be reported by nursing homes and hospitals than from other institutions

\begin{tabular}{rr}
\multicolumn{2}{c}{ NSW population } \\
Male & $50 \%$ \\
$<5$ & $7 \%$ \\
$5-24$ & $28 \%$ \\
$25-64$ & $52 \%$ \\
$65+$ & $13 \%$ \\
Rural $^{*}$ & $42 \%$
\end{tabular}

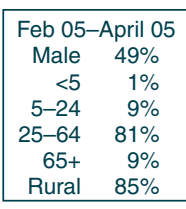

Rural $85 \%$
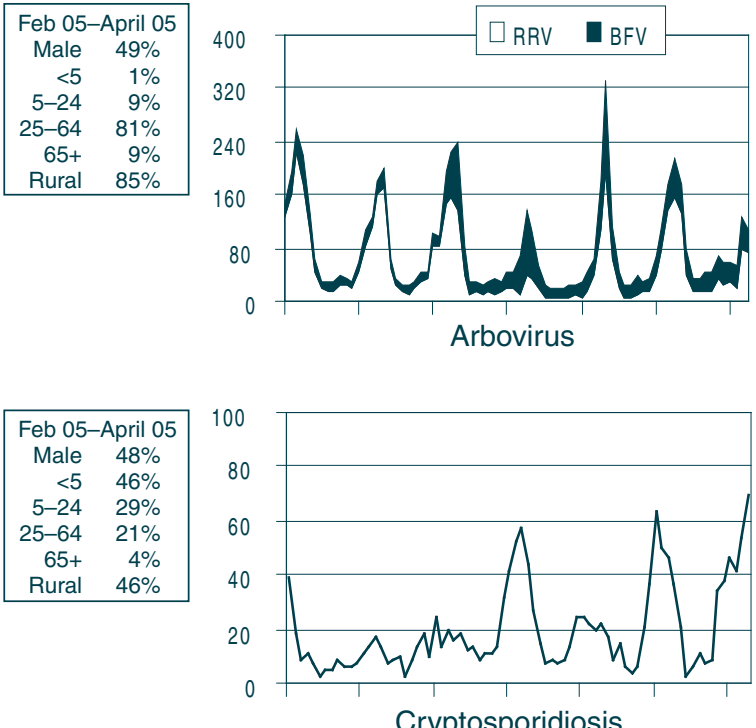

\begin{tabular}{|cc|}
\hline \multicolumn{2}{|c|}{ Feb 05-April 05 } \\
Male & $88 \%$ \\
$<5$ & $0 \%$ \\
$5-24$ & $26 \%$ \\
$25-64$ & $74 \%$ \\
$65+$ & $0 \%$ \\
Rural & $18 \%$ \\
\hline
\end{tabular}
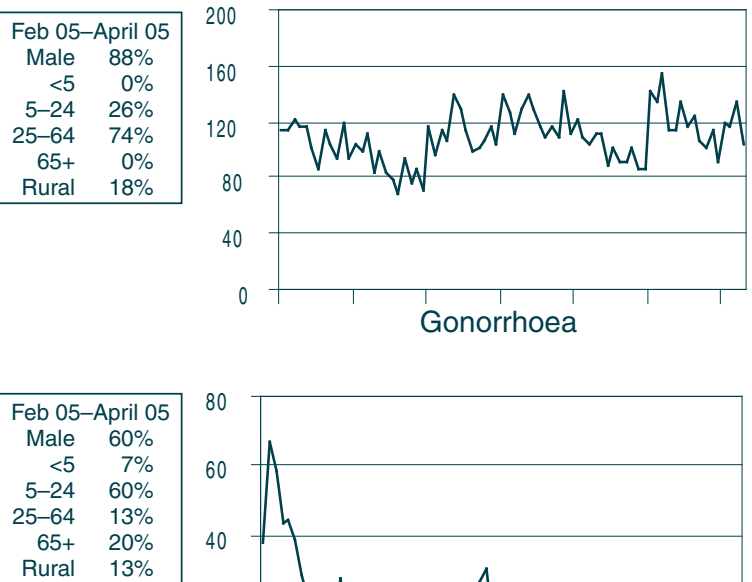

Rural $13 \%$

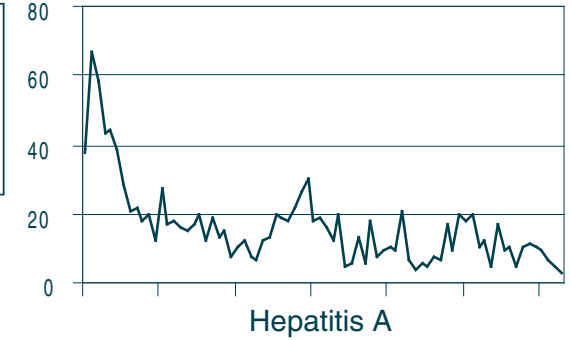

\begin{tabular}{|lr|}
\hline \multicolumn{2}{|c|}{ Feb 05-April 05} \\
All outbreaks & 6 \\
Nursing homes & 3 \\
Hospitals & 0 \\
Child care & 3 \\
Schools & 0 \\
Other & 0 \\
\hline
\end{tabular}

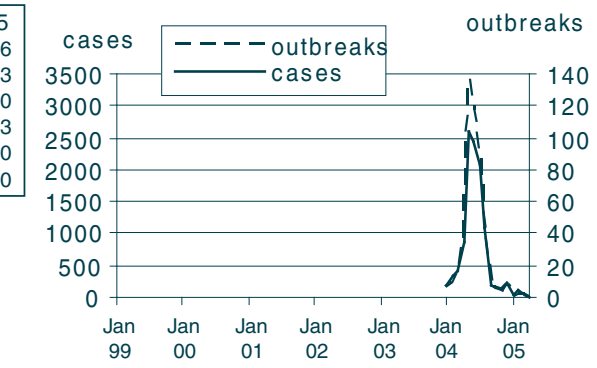

Gastroenteritis outbreaks in institutions

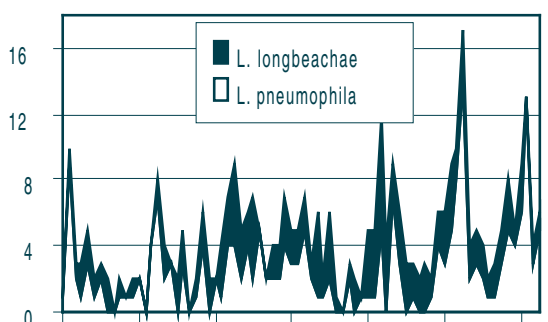

Legionellosis

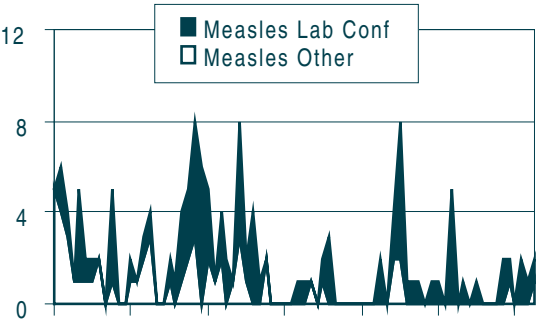

Measles

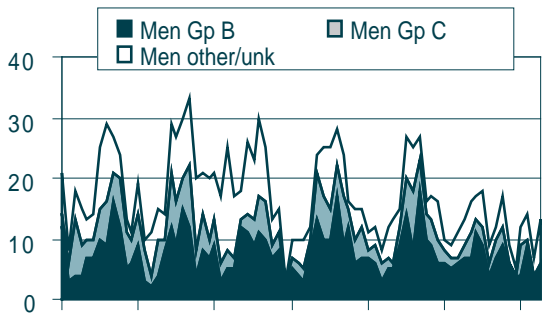

Meningococcal disease

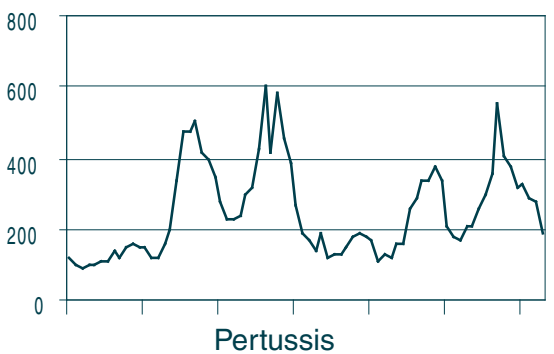
$<5 \quad 44 \%$

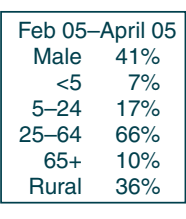

Feb 05-April 05 Male $53 \%$ $\begin{array}{rr}5-24 & 27 \% \\ 25-64 & 23 \%\end{array}$

$\begin{array}{rr}25-64 & 23 \% \\ 65+ & 6 \%\end{array}$ Rural $41 \%$

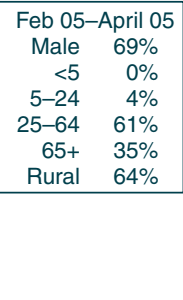

\begin{tabular}{cc}
\multicolumn{2}{|c|}{ Feb 05-April 05 } \\
Male & $60 \%$ \\
$<5$ & $0 \%$ \\
$5-24$ & $40 \%$ \\
$25-64$ & $60 \%$ \\
$65+$ & $0 \%$ \\
Rural & $0 \%$
\end{tabular}

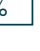

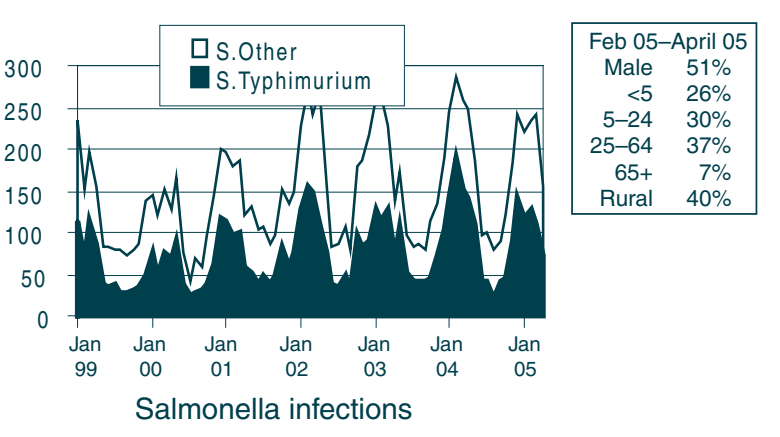

Salmonella infections 


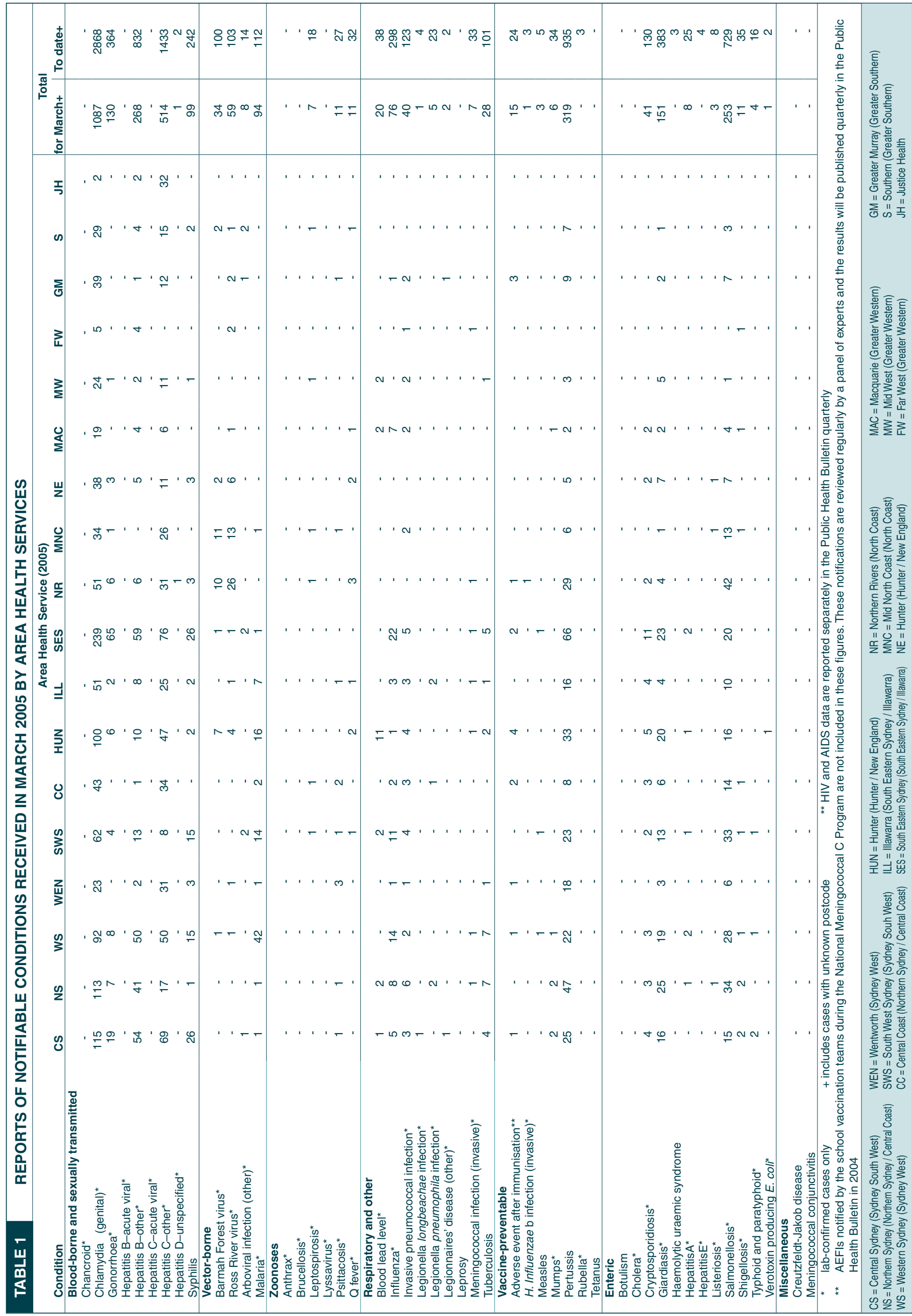




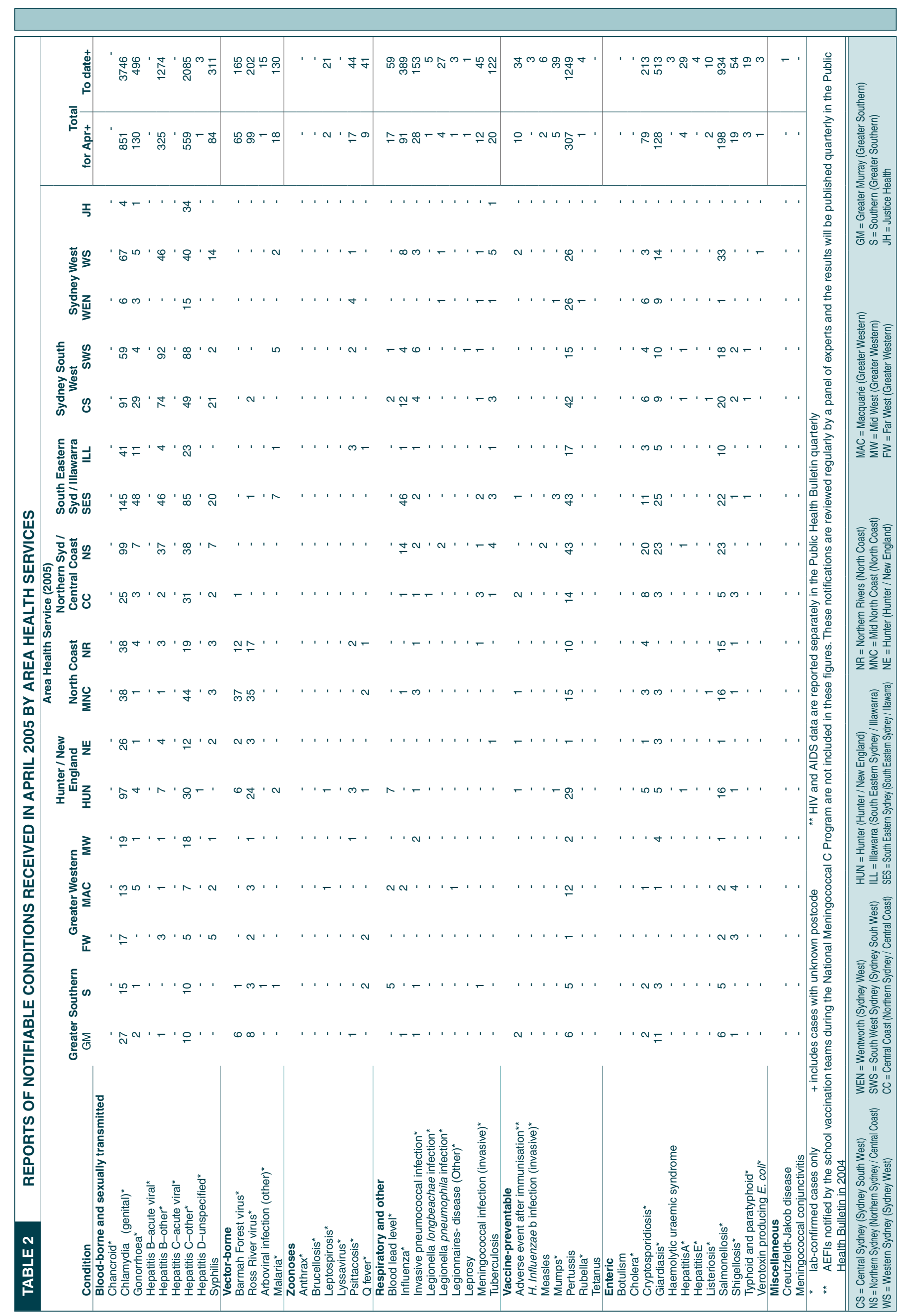

